\title{
Medizinische Qualitätsarbeit - eine Bestandesaufnahme in Kleinporträts (1)
}

Was einzelnen Ärzten einen Seufzer entlockt, erfüllt andere Berufskolleginnen mit Stolz und Befriedigung: Qualitätsarbeit ist in der Medizin mittlerweile eine Realität - mit Entwicklungspotential. So ist es auch der Arbeitsgruppe Qualität der FMH (AGQ-FMH) ein Anliegen, die FMHMitglieder über bestehende Qualitätsinitiativen aus der Schweizer Medizinlandschaft zu informieren und sie dafür zu begeistern. $\mathrm{Zu}$ diesem Zweck stellt die AGQ-FMH in jeweils fünf aufeinanderfolgenden Ausgaben der Schweizer Ärzte- zeitung in aller Kürze und in übersichtlicher Form jene Qualitätsinitiativen vor, die ihr Referenten aus allen Fachgebieten und Arbeitsbereichen (stationär/ambulant) seit 2006 präsentiert haben. Zeitgleich werden diese Kurzporträts mit weiterführenden Informationen auf der Webseite der FMH-Abteilung Daten, Demographie und Qualität aufgeschaltet. Den Auftakt machen im vorliegenden Heft das Europäische Praxisassessment EPA und EQUAM, die Externe Qualitätsarbeit in der Medizin.
Beat Künzi

Korrespondenz: Dr. Beat Künzi

Swisspep Institut für Qualität und Forschung im Gesundheitswesen Mattenstrasse 9 / Postfach

CH-3073 Gümligen

Tel. 0319516969

Fax 0319516977

swisspep@hin.ch

www.swisspep.ch

\section{Europäisches Praxisassessment EPA}

\section{Beschreibung}

- EPA ist ein System zur Einführung und Weiterentwicklung des Qualitätsmanagements (QM) in Hausarzt- und Facharztpraxen sowie in HMO und Ärztenetzwerken. EPA unterstützt die Leistungserbringer bei der kontinuierlichen Verbesserung ihrer Tätigkeit und sichert damit den Patienten eine effiziente Betreuung für eine optimale Gesundheit.

- EPA setzt sich zusammen aus innovativen QM-Systemen, basierend auf den 20012005 international entwickelten und wissenschaftlich validierten EPA-Qualitätsindikatoren.

- In der Schweiz bildet EPA die Grundlage für eine EQUAM-Zertifizierung (optional; 2006 von der Schweizerischen Akkreditierungsstelle SAS des Staatssekretariats für Wirtschaft SECO akkreditiert).

Die aktuell 225 EPA-Qualitätsindikatoren decken einen umfassenden Bereich qualitätsrelevanter Aspekte ärztlicher Versorgung ab. Sie erlauben Praxisteams wie Ärztenetzwerken, sich mit den Besten direkt zu vergleichen oder dann - anhand dieser «Benchmarks» - konkrete Verbesserungen zu erkennen. Die EPA-Qualitätsindikatoren haben folgende Eigenschaften:

- sie sind selbsterklärend für Ärzte;
- spezielle QM-Schulungen sind nicht notwendig;

- sie basieren auf wissenschaftlichen Methoden und modular erweiterbaren Instrumenten (z. B. Zuweiserbefragung);

- sie bringen nachweislich Verbesserungen von Behandlungs- und Arbeitsabläufen, ohne unnötigen administrativen Aufwand;

- sie ermöglichen eine national und international vergleichende Versorgungsforschung.

Weitere EPA-Elemente zur Schaffung von lernenden Organisationen, die komplexe Versorgungsprozesse nachhaltig verbessern:

- Einbezug des ganzen Teams;

- kontinuierliche Unterstützung durch eine internetbasierte Visotool ${ }^{\circledR}$-Benchmark-Datenbank und Materialien sowie Workshops;

- Selbst- und Fremdevaluation der Performance durch Teammitglieder, Patienten und durch eine speziell ausgebildete EPA-Visitorin, die dann direkt die Teamsitzung zur Definition von internen Qualitätsprojekten leitet.

Vom Zeitpunkt der ersten Kontaktnahme über die Vorbereitung des Mitarbeiter- und Patientenbefragung bis zur Visitation mit Schlussbesprechung nimmt ein EPA 6-8 Wochen in Anspruch (s. auch Aufwand und Kosten). 


\section{Geeignet für}

- Grundversorger- und Facharztpraxen;

- Pädiater;

- Psychiater (in Pilotphase).

\section{Aufwand und Kosten}

Der Aufwand für ein EPA wird von $2 / 3$ der befragten Praxen als realistisch und verhältnismässig eingestuft: etwa $1 \frac{1}{2}$ Arbeitstag je für den verantwortlichen Arzt und eine MPA zur Vorbereitung, Team- und Patientenbefragung sowie Visitation (inkl. der etwa 90-minütigen Teamsitzung); dieser Zeitaufwand bleibt für alle Praxisgrössen etwa gleich; einzig zur Zertifizierung von HMO bzw. Netzwerken kommen noch etwa 2 Stunden dazu (für die Überprüfung der MC-Indikatoren des EQUAM-B-Teils).

\section{Kosten (Leistungen gemäss Preisliste)}

- Einzel- und Doppelpraxen: Fr. 2685.- inkl. MwSt. (= Fr. 2495.35 netto);

- Praxen mit 3-5 Ärzten: Fr. 4030.- inkl. MwSt. (= Fr. 3745.35 netto);

- Praxen mit über 5 Ärzten: Fr. 6290.- inkl. MwSt. (= Fr. 5845.70 netto).
Felix Roth

Korrespondenz: Dr. Felix Roth

Präsident und Geschäftsführer

EQUAM-Stiftung

Therwilerstrasse 3

CH-4054 Basel

Tel. 0612848288

office@equam.ch

www.equam.ch

www.equam.org

\section{EQUAM - Externe Qualitätssicherung in der Medizin}

\section{Beschreibung}

- Die EQUAM-Stiftung fördert die externe, unabhängige Qualitätssicherung in der ambulanten medizinischen Versorgung.

- Sie gewichtet ausgewählte EPA-Indikatoren und setzt Minimalstandards.

- Für HMO-Praxen und Ärztenetzwerke werden zusätzlich Managed-Care-Prozessindikatoren formuliert.

- Für Arztpraxen entwickelt EQUAM diagnosespezifische Minimalstandards für die klinische Outcome- und Performancequalität.

Die nichtgewinnorientierte EQUAM-Stiftung ist von der Schweizerischen Akkreditierungsstelle des seco als Zertifizierungsstelle für Qualitätssicherung in Arztpraxen akkreditiert. Sie vereinigt im Stiftungsrat Vertreter von FMH, SUVA, santésuisse, Patientenorganisationen (SPA, DVSP), Konsumentenschutz sowie Parlamentarier und Fachexperten.

Die EQUAM-Stiftung gewichtet für die Zertifizierung von Arztpraxen ausgewählte EPA-Indikatoren anhand von Punktzahlen und definiert andere EPA-Indikatoren als Minimalstandards (Modul A). So wird z.B. die Einführung eines Fehlermanagements mit Punkten belohnt und der Schutz der Patientenakten vor Einsicht Dritter oder die Durchführung einer Patientenbefragung gefordert. Für HMO-Praxen und Ärztenetzwerke werden zusätzliche Managed-Care-Prozessindikatoren gemessen, z.B. Qualitätszirkel, Disease Management, Guidelines (Modul B). Arztpraxen können zudem in klinischer Ergebnisqualität zertifiziert werden (Modul C). Die der Ergebnisqualität zugrundeliegenden EQUAM-
Standards betreffen jeweils eine Diagnose (z. B. arterielle Hypertonie, Diabetes mellitus) und orientieren sich an der bestmöglichen wissenschaftlichen Evidenz.

Das EQUAM-Zertifikat wird jeweils für drei Jahre vergeben. In dieser Zeit muss eine kontinuierliche Qualitätsarbeit geleistet werden; dies z.B. durch die Definition von Zielen und entsprechende Arbeit an diesen Zielen oder durch das Realisieren des PDCA-Zyklus (Plan-DoCheck-Act-Zyklus).

\section{Geeignet für}

- Einzelpraxen;

- HMO-Praxen;

- Ärztenetzwerke.

\section{Aufwand und Kosten}

\section{Einzel- und Doppelpraxis}

Aufwand: EPA (Selbstdeklaration: 2 Stunden; Patientenbefragung, Interview: 2 Stunden; Audit vor Ort mit MPA, Schlussbesprechung: 1 Stunde), Zieldefinition und Arbeit an den Zielen.

Kosten: Zertifizierungsgebühr Fr. 500.-/Jahr (exkl. MwSt.) plus Kosten für EPA.

\section{Ärztenetzwerk (Beispiel mit 30 Praxen)}

Aufwand: Dokumentation der Aktivitäten wie Qualitätszirkel, Disease Management, Guidelines und Bereitstellung dieser Unterlagen in der Geschäftsstelle, Besprechung anlässlich des Audits mit dem Netzleiter: 2 Stunden.

Kosten: zusätzliche Zertifizierungsgebühr Fr. 4750.-/Jahr (exkl. MwSt.) plus Auditkosten Fr. 2100.- (exkl. MwSt.). 